\title{
Analytical Solutions of One-Dimensional Convection-Diffusion Problems
}

\author{
Hatıra Günerhan* \\ Department of Mathematics, Faculty of Education, Kafkas University, Kars, Turkey \\ *Corresponding author: gunerhanhatira@gmail.com
}

Received September 01, 2018; Revised October 04, 2018; Accepted November 05, 2018

\begin{abstract}
This paper first describes RDTM and then uses Reduced RDTM to compute an approximate solution of the one-dimensional convection-diffusion problems. The method provides the solution quickly in the form of a convergent series. Our method reduces the computational difficulties existing in the other traditional methods. To show the efficacy of the results, three examples are considered.
\end{abstract}

Keywords: reduced differential transform method (RDTM), nonhomogeneous convection-diffusion problem, one-dimensional convection-diffusion problems

Cite This Article: Hatıra Günerhan, "Analytical Solutions of One-Dimensional Convection-Diffusion Problems." Turkish Journal of Analysis and Number Theory, vol. 6, no. 6 (2018): 152-154. doi: 10.12691/tjant-6-6-1.

\section{Introduction}

We consider the following one-dimensional convection-diffusion equation

$$
\begin{gathered}
\frac{\partial u(x, t)}{\partial t}+\propto \frac{\partial u(x, t)}{\partial x}+\beta u(x, t)-\gamma \frac{\partial^{2} u(x, t)}{\partial x^{2}}=f(x, t) \\
\mathrm{m} \leq \mathrm{x} \leq \mathrm{n}, t \geq 0 \\
u(m, t)=k_{1}(x), u(n, t)=k_{2}(x), t \geq 0 \\
u(x, 0)=h(x), \mathrm{m} \leq \mathrm{x} \leq \mathrm{n}
\end{gathered}
$$

Where $\alpha, \beta$ and $\gamma$ are known constant coefficients, $k_{1}(x)$ and are known continuous functions.

The convection-diffusion equation is a merging of diffusion and convection equations, and describes the physical phenomena in which particles, energy, or other physical quantities are transmitted to the physical system due to the two processes of diffusion and convection.

In this study, firstly we describe the RDTM and then apply the RDTM to investigate the solution of the onedimensional Convection-diffusion equation.

In recent years, some numerical solution have been developed to solve these types of convection-diffusion problems. Likes: the adaptive spline function approximation [1], several finite element methods [2], the finite difference approximation [3], cubic B-spline quasi-interpolation [4], restrictive taylor's approximation [5], exponential B-spline collocation method [6]. Exponential B-splines [7], weighted finite difference [8], meshless method [9], implicit method [10], redefined cubic B-splines collocation method [11]. In 2009, the reduced differential transform method (RDTM) was introduced by Keskin [12] to solving solve various partial differential equations and fractional nonlinear partial differential equations. The technique of reduced differential transforms is a repetitive procedure for the solution of a Taylor series differential equations. This method reduces the size of the computational work and can be easily applied to many physical problems.

This paper has been organized as follows: Section 2 deals with the analysis of the method. In Section 3, we apply the RDTM to solve three types of one-dimensional Convection-Diffusion Problem. Conclusions are given in Section 4.

\section{Reduced Differential Transform Method (RDTM)}

Consider a function of two variables $f(x, t)$, and suppose that it can be represented as a product of two single-variable $f(x, t)=g(x) h(t)$. the function

$f(x, t)$ can be represented as

$$
\begin{aligned}
& \mathrm{f}(\mathrm{x}, \mathrm{t})=\left(\sum_{i=0}^{\infty} \mathrm{G}(i) x^{i}\right)\left(\sum_{j=0}^{\infty} \mathrm{H}(j) t^{j}\right) \\
& =\sum_{i=0}^{\infty} \sum_{j=0}^{\infty} \mathrm{G}(i) \mathrm{H}(j) x^{i} t^{j}=\sum_{k=0}^{\infty} F_{k}(x) t^{k},
\end{aligned}
$$

Where $F_{k}(x)$ is called $t$-dimensional spectrum function of $f(x, t)$. The basic definitions and fundamental operations of the two-dimensional reduced differential transform method are introduced are defined in [13] as follows:

Definition 2.1. Assume $f(x, t)$ is an analytic function in the domain of interest. The reduced differential transform of $f(x, t)$ is defined as 


$$
F_{k}(x)=\frac{1}{\mathrm{k} !}\left[\frac{\partial^{k}}{\partial t^{k}} \mathrm{f}(\mathrm{x}, \mathrm{t})\right]_{\mathrm{t}=0},
$$

Definition 2.2. The differential inverse transform of $F_{k}(x)$ is defined as follows:

$$
f(x, t)=\sum_{k=0}^{\infty} U_{k}(x) t^{k} .
$$

By inserting equation (2.2) in (2.3), we obtain

$$
f(x, t)=\sum_{k=0}^{\infty} \frac{1}{\mathrm{k} !}\left[\frac{\partial^{k}}{\partial t^{k}} u(x, t)\right]_{\mathrm{t}=0} t^{k} .
$$

Some basic properties of the two dimensional reduced differential transform are presented in Table 1 below.

Table 1. The Operations for the reduced differential transform method

\begin{tabular}{|l|l|}
\hline Original function & Transformed function \\
\hline$f(x, t)=g(x, t) \pm h(x, t)$ & $F_{k}(x)=G_{k}(x) \pm H_{k}(x)$ \\
\hline$f(x, t)=e^{\gamma t}$ & $F_{k}(x)=\frac{\gamma^{k}}{k !}$ \\
\hline$f(x, t)=\frac{\partial^{m}}{\partial t^{m}} g(x, t)$ & $F_{k}(x)=\frac{(k+m) !}{k !} G_{k+m}(x)$ \\
\hline$f(x, t)=g(x, t) h(x, t)$, & $F_{k}(x)=\sum_{l=0}^{k} G_{l}(x, y) H_{k-l}(x)$ \\
\hline$f(x, t)=\frac{\partial^{m}}{\partial x^{m}} g(x, t)$ & $F_{k}(x)=\frac{\partial^{m}}{\partial x^{m}} G_{k}(x)$ \\
\hline$f(x, t)=x^{a} t^{d}$ & $\begin{array}{l}F_{k}(x)=x^{a} \delta(k-d) \\
=\left\{\begin{array}{c}x^{a}, \quad k=d \\
0, k \neq d\end{array}\right.\end{array}$ \\
\hline$f(x, t)=\frac{k}{\partial x^{a} \partial t^{d}} g(x, t)$ & $F_{k}(x)=\frac{\partial^{a}}{\partial x^{a}} \frac{(k+d) !}{k !} G_{k+d}(x)$ \\
\hline
\end{tabular}

\section{Numerical Example}

In this section, we used the basic definitions (in section 2) of the two-dimensional reduced differential transform method for solving three examples of the one-dimensional Convection-diffusion equations.

Example 1. We consider the following one-dimensional convection-diffusion problem

$$
\begin{aligned}
\frac{\partial f}{\partial t}-\frac{\partial^{2} f}{\partial x^{2}} & =2 t \sin (2 \pi x)+4 \pi^{2} t^{2} \sin (2 \pi x), \\
0 & \leq t, x \leq 1,
\end{aligned}
$$

with the boundary conditions and the initial condition

$$
\begin{aligned}
& f(0, t)=0, f(1, t)=0,0 \leq t \leq 1, \\
& f(x, 0)=0,0 \leq x \leq 1
\end{aligned}
$$

by using the basic properties of RDTM in equations (3.1) and (3.2), we obtain the following relations

$$
\begin{aligned}
& (k+1) F_{k+1}(x)-\frac{\partial^{2}}{\partial x^{2}} F_{k}(x) \\
& =2 \delta(k-1) \sin (2 \pi x)+4 \pi^{2} \delta(k-2) \sin (2 \pi x) .
\end{aligned}
$$

From initial condition (3.2), we have

$$
F_{0}(x)=0 \text {. }
$$

Using Eq. (3.4) in Eq. (3.3), we get the following $U_{k}(x)$ values,

$$
\begin{aligned}
& F_{1}(x)=0, F_{2}(x)=\sin (2 \pi x), \\
& U_{i}(x)=0, i=3,4,5, \ldots
\end{aligned}
$$

By using the differential inverse reduced transform of $U_{k}(x, y)$

$$
\begin{aligned}
& f(x, t)=\sum_{k=0}^{\infty} F_{k}(x) t^{k} \\
& =F_{0}(x)+F_{1}(x) t+F_{2}(x) t^{2}+F_{3}(x) t^{3}+\ldots
\end{aligned}
$$

we get

$$
f(x, t)=\sin (2 \pi x) t^{2} .
$$

We obtain the exact solution of equation (3.1).

Example 2. We consider the convection-diffusion

$$
\frac{\partial f}{\partial t}+\frac{\partial f}{\partial x}=\frac{\partial^{2} f}{\partial x^{2}}, 0 \leq t, x \leq 1,
$$

and the boundary conditions

$$
f(0, t)=e^{\left(\frac{3}{4}\right) t}, f(1, t)=e^{\left(\frac{-1}{2}\right)\left(\frac{3}{4}\right) t},
$$

subject to the initial conditions

$$
f(x, 0)=e^{\left(\frac{-x}{2}\right)} .
$$

By using the basic properties of RDTM in equation (3.8), we obtain the following relations

$$
(k+1) F_{k+1}(x)+\frac{\partial}{\partial x} F_{k}(x)=\frac{\partial^{2}}{\partial x^{2}} F_{k}(x) .
$$

Taking the differential transform of Eqs.(3.10), we write

$$
F_{0}(x)=e^{\left(\frac{-x}{2}\right)}
$$

Using Eq. (3.12) in Eq. (3.11), we get the following $U_{k}(x)$ values

$$
\begin{aligned}
& F_{1}(x)=\frac{3}{4} e^{\left(\frac{-x}{2}\right)}, F_{2}(x)=\frac{9}{32} e^{\left(\frac{-x}{2}\right)}, \\
& F_{3}(x)=\frac{9}{128} e^{\left(\frac{-x}{2}\right)}, F_{4}(x)=\frac{27}{2048} e^{\left(\frac{-x}{2}\right)}, \\
& F_{5}(x)=\frac{81}{327680} e^{\left(\frac{-x}{2}\right)}, \ldots
\end{aligned}
$$

the exact solution of the equation (3.8) will assume the following form:

$$
f(x, t)=\sum_{k=0}^{\infty} F_{k}(x) t^{k}=e^{\left(\frac{-x}{2}\right)\left(\frac{3}{4}\right) t} .
$$

Example 3. Consider the convection-diffusion problem

$$
\frac{\partial f}{\partial t}+\frac{\partial f}{\partial x}+2 f=\frac{\partial^{2} f}{\partial x^{2}}+\left(x^{2}+2 x-2\right) e^{-t}, 0 \leq t, x \leq 1,
$$


with the initial condition

$$
f(x, 0)=x^{2},
$$

with the boundary condition:

$$
f(0, t)=0, f(1, t)=e^{-t}
$$

by using the basic properties of RDTM in equation (3.15) and (3.16), we obtain the following relations

$$
\begin{aligned}
& (k+1) F_{k+1}(x)+\frac{\partial}{\partial x} F_{k}(x)+2 F_{k}(x) \\
& =\frac{\partial^{2}}{\partial x^{2}} F_{k}(x)+\left(x^{2}+2 x-2\right) \frac{(-1)^{k}}{k !}
\end{aligned}
$$

and

$$
u_{0}(x)=x^{2}
$$

Using Eq. (3.18) in Eq. (3.19), we get the following $U_{k}(x)$ values

$$
\begin{aligned}
& u_{1}(x)=-x^{2}, u_{2}(x)=\frac{1}{2} x^{2}, u_{3}(x)=-\frac{1}{6} x^{2}, \\
& u_{4}(x)=\frac{1}{24} x^{2}, u_{5}(x)=-\frac{1}{120} x^{2}, \\
& u_{6}(x)=\frac{1}{720} x^{2}, \ldots
\end{aligned}
$$

By using the differential inverse reduced transform of $U_{k}(x)$, we get

$$
\begin{aligned}
u(x, t)=\sum_{k=0}^{\infty} U_{k}(x) t^{k} \\
=U_{0}(x, y)+U_{1}(x, y) t^{1} \\
+U_{k}(x, y) t^{2}+U_{k}(x, y) t^{3}+\ldots \\
=x^{2}\left(1-t+\frac{t^{2}}{2 !}-\frac{t^{3}}{3 !}+\frac{t^{4}}{4 !}-\frac{t^{5}}{5 !}+\frac{t^{6}}{6 !}-\frac{t^{7}}{7 !}+\ldots\right),
\end{aligned}
$$

by using the closed form in the solution of (3.21), we obtain the following exact solution

$$
u(x, y, t)=x^{2} e^{-t} .
$$

\section{Conclusion}

In this work, we used reduced differential transform method (RDTM) to compute an approximate solution of the one-dimensional convection-diffusion problems. The method provides the solution in the form of a rapidly convergent series. Three explanatory examples are given to represent the efficacy of the obtained results.

\section{References}

[1] Jain, M.K., Tariq, A., Numerical solution of stiff and convectiondiffusion equations using adaptive spline function approximation, Applied Mathematical Modelling,7(1), 57-62, 1983.

[2] Codina, R., Comparison of Some Finite-Element Methods for Solving the Diffusion-Convection-Reaction Equation, Computer Methods in Applied Mechani - gineering, 156(1), 185-210,1998.

[3] Salkuyeh, D.K.. On the finite difference approximation to the convection diffusion equation, Appl. Math. Comput, 179, 79-86, 2006.

[4] Aminikhah, H., and Alavi, J., Numerical Solution of ConvectionDiffusion Equation Using Cubic B-Spline Quasi-Interpolation, Thai Journal of Mathematics, 14 (3), 599-613, 2016.

[5] Ismail, H.N.A., Elbarbary, E.M.E., and Salem, G.S.E., Restrictive Taylor's approximation for solving convection-diffusion equation, Appl. Math. Comput., 147, 355-363, 2004.

[6] Dehghan, M., On the Numerical solution of Dimensional Convection-Diffusion Equation, Mahtical Problems in Engineering, 2005(1), 61-74, 2005.

[7] Mohammadi, R., Exponential B-Spline Solution of ConvectionDiffusion Equations, Applied Mathematics, 4, 933-944, 2013.

[8] Dehgan, M., Weighted Finite Difference Techniques for the OneDimensional Advection Diffusion Equation, Applied Mathematics and Computation, 147(2), 307-319, 2004.

[9] LD, S., ZW, J., TS, J., Numerical Method for One-Dimensional Convection-diffusion Equation Using Radical Basis Functions, J Phys Math, 6(1), 136, 2015.

[10] Ravi Kanth, A.S.V., a and Deepika, Numerical Solutions for Convection-Diffusion Equation through Non-Polynomial Spline, MATEC Web of Conferences 57, 05004, 2016.

[11] Mittal, R.C. and Jain, R.K., Redefined Cubic B-Splines Collocation Method for Solving Convection-D usion Equations, Applied Mathematical Modelling, 36(11), 5555-5573, 2012,

[12] Keskin, Y., Ph.D Thesis, Selcuk University, Turkey, 2010.

[13] Benhammouda, B., Vazquez-Leal, H., and Sarmiento-Reyes A., Modified Reduced Differential Transform Method for Partialm Differential-Algebraic Equations, SpringerPlus, 4(1), 1-9, 2015. 